\title{
DEVELOPMENT OF A SCINTILLATING FIBER TRACKER FOR THE SSC
}

Final Technical Report

for the period July 1, 1991 - June 30, 1992

Raymond A. Lewis

\author{
Pennsylvania State University \\ University Park, PA 16802
}

October 1992

Prepared for

THE U.S. LEPARTMENT OF ENERGY

AGREEMENT NO. DE-FG02-91ER40653

\begin{abstract}
NOTICE
This report was prepared as an account of work sponsored by the United States government. Neither the United States nor the Department of Energy, nor any of their employees, nor any of their contractors, subcontractors, or their employees, makes any warranty, express or implied, or assumes any legal liability or responsibility for the accuracy, completeness, or usefulness of any information, apparatus, product or process disclosed or represents that its use would not infringe privately-owned rights.
\end{abstract}




\author{
T.A. Armstrong, R.A. Lewis, G.S. Mitchell, R.S. Moore, \\ J.E. Passaneau and G.A. Smith \\ Penn State University \\ October 7, 1992
}

\begin{abstract}
The Fiber Tracking Group (FTG) of the Solenoidal Detector Collaboration (SDC) is developing a high rate tracking subsystem appropriate for the SDC detector based on scintillating fiber (SciFi) technology. In the past two years a major upgrade in the design was conceived, which reduces the material and cost of the tracker and improves the effectiveness of the tracking system as a trigger element. The results of simulations of the performance of the tracker at Penn State have been very important in keeping the Scifi technology as an attractive option for a tracking system for the SDC collaboration.

In the past year an array of scintillating fiber elements has been evaluated in a test beam at Brookhaven. Past experience of Penn State personnel at Brookhaven proved valuable in making the test beam runs a success.
\end{abstract}

\title{
UPGRADE OF TRACKER DESIGN
}

The original design of the SciFi tracker is summarized in a paper presented at the Fort Worth Symposium [Appendix A]. The basic design of the tracker consisted of four superlayers, each containing two sublayers of four layers of fibers separated by $5 \mathrm{~cm}$.

Later, a suggestion was made for improving the porformance of the scintillating fiber tracker. The sublayers were rearranged with pairs of closepacked fiber ribbons separated by $1.6 \mathrm{~cm}$. The doublet pair configuration provides local pattern recognition for distinguishing high $p_{t}$ tracks from background. The new configuration with six doublet pair superlayers shown in Figure 1 contains less than half the number of fibers as in the original design. The reduced fiber count results in a savings in cost and in the material thickness which creates unwanted secondary interactions such as Bremsstrahlung.

Simulations at Penn State showed that the doublet pair configuration results in an order of magnitude improvement in background rejection at the trigger level. The local pattern recognition reduces by a factor of five the number of hits which must be linked to recognize high $p_{t}$ tracks. This improvement allows a simpler trigger, involving only the three inner superlayers, to be used effectively.

The advantage of using the inner three superlayers is that triggering coverage can be extended to large rapidities, $|\eta|-2.3$, simply by lengthening the fibers in these layers. The rapidity coverage of the outer superlayers is limited by the four meter length of the tracking volume. 
Coverage of the large rapidity region is a challenging task. An alternative is to use gas microstrips in this region, which is more expensive and unproven at this time. The use of scintillating fibers solves the problem fust by extending the length of the fibers. This solution works for luminosities up to several times design luminosity. At higher luminosity the occupancy of the inner three superlayers results in a large combinatorial background of fake stiff track triggers, requiring the use of outer superlayers with reduced rapidity coverage.

A paper documenting the simulated evaluation of various scintillating fiber tracker designs is being prepared at Penn State for publication [Appendix B]. Portions of the paper which document the evaluation of the latest tracker design are included in the SDC Technical Design Report (SDC-92-201, April 1, 1992).

\section{TEST BEAM ACTIVITIES}

A crucial consideration in making the doublet layer concept work is light yield and hence efficiency of the fibers. A sample of 16 fibers was evaluated in the FNAL muon test beam in January 1992. This run demonstrated that scintillating fibers read out by VLPC devices could be operated in a test beam environment, and produce tracking resolution consistent with the granularity of the fibers. A report on these test beam results has been submitted for publication in Nuclear Instruments and Methods (Appendix C).

Subsequently, an array of 126 fibers was tested in the A2 test beam at Brookhaven during June-July, 1992, to evaluate brightness as well as systems issues. R. Lewis was the spokesman for the test beam activities. The detector elements consisted of 4 meters of scintillating fiber and 6 meters of clear waveguide, similar to the lengths to be used in the SDC tracker. The photodetectors were Visible Light Photon Counters (VLPC's) called HISTE-III, designating the third version of such devices developed by Rockwell International Corp. These VLPC's have a photoelectric efficiency around 608, lower than the 808 which will ultimately be used in the tracker.

The signals detected by the VLPC's from minimum ionizing beam particles are typically several photoelectrons, amplified by a factor of 10,000 . Measuring these low-level signals in the environment of the Brookhaven experimental hall was a challenge. Thanks to previous experience in the BNL environment, J. Passaneau (Penn State) was very effective in arranging electronics grounding to suppress background noise well below the level of one photoelectron signals.

The layout of the fibers is illustrated in Figure 2. The 12 layers are arranged in close-packed doublets. A pair of doublets spaced $16 \mathrm{~mm}$ apart constitutes a superlayer. The three superlayers are separated by $160 \mathrm{~mm}$. Each fiber consists of 4 meters of scintillator $0.85 \mathrm{~mm}$ diameter, connected to a VLPC through 6 meters of light guide.

Beam tracks detected in the first five doublet layers define which $f i$ bers in the sixth doublet should be hit. Figures 3 and 4 show pulse height spectra for the following:

Figure 3 - fiber 109, beam near scintillator - waveguide junction, Figure 4 - fiber 118, beam 4 meters from junction (10 meters from the VLPC). 
The following table summarizes our interpretation of Figs. 3 and 4 .

Beam at

《No. photoelectrons>

r.m.s. spread

single fiber eff. waveguide-scintillator

interface

$2.36 \pm 0.08$

$2.01 \pm 0.08$

$81 \pm 28$ far end

of scintillator

$1.14 \pm 0.07$

$1.14 \pm 0.07$

The efficiency is defined as the fraction of beam particles with a pulse height above 0.66 photoelectrons, the minimum between the pedestal (noise, 0 p.e. peak) and one p.e. peaks (see Figs. 3,4).

The curves on Figs. 3 and 4 are Poisson distributions, calculated assuming the above means and normalized to the total number of counts. The nonPoisson character of the data is due to the fact that all beam particles do no traverse equal path lengths in the fibers because of their circular cross sections. In addition, the fibers have a dead region of $0.10 \mathrm{~mm}$ between them consisting of glue and cladding material, resulting in a geometrical efficlency of 898. This represents an upper limit efficiency expected in these measurements. The observed efficlencies are consistent with this value within errors, correcting the far end measurements for light absorption over the $4 \mathrm{~m}$ length of the fiber using an attenuation length of $5.4 \mathrm{~m}$.

Since the fibers are close packed as shown in Figure 2, the pulse heights in the two fibers in a doublet hit by a beam particle should be correlated. Ignoring the benefits of this correlation, the efficiency of a doublet can be estimated as follows:

$$
\begin{array}{ll}
\text { near end: } & \text { eff(doublet) }=1-(1-0.81)^{2}-968 \\
\text { far end: } & \text { eff }(\text { doublet })=1-(1-0.58)^{2}=828
\end{array}
$$

By comparison, the observed efficiency for finding a hit in the sixth doublet is 978 for beam particles at near end and 848 for beam particles at the far end of the fiber. Both values are in excellent agreement with (1).

Three improvements in the design are expected to bring the doublet efficiency up to the 978 requirement outlined in the Technical Design Report. A new version of the VLPC, HISTE-IV, will combine the high quantum efficiency (85\%) of the original SSPM (solid state photomultiplier) with the infrared insensitivity of the HISTE-III devices used in the test run. This will increase the photoelectron yields by a factor of 1.5. The fiber diameters will be increased from 0.85 to $0.95 \mathrm{~mm}$, resulting in a factor of 1.25 increase, due to longer particle path lengths and to a reduced number of reflections as light propagates through the fiber. In addition, the noise level will be reduced by using faster pre-amplifiers, so the cut between the pedestal (noise) and the one photoelectron peak can be lowered. 


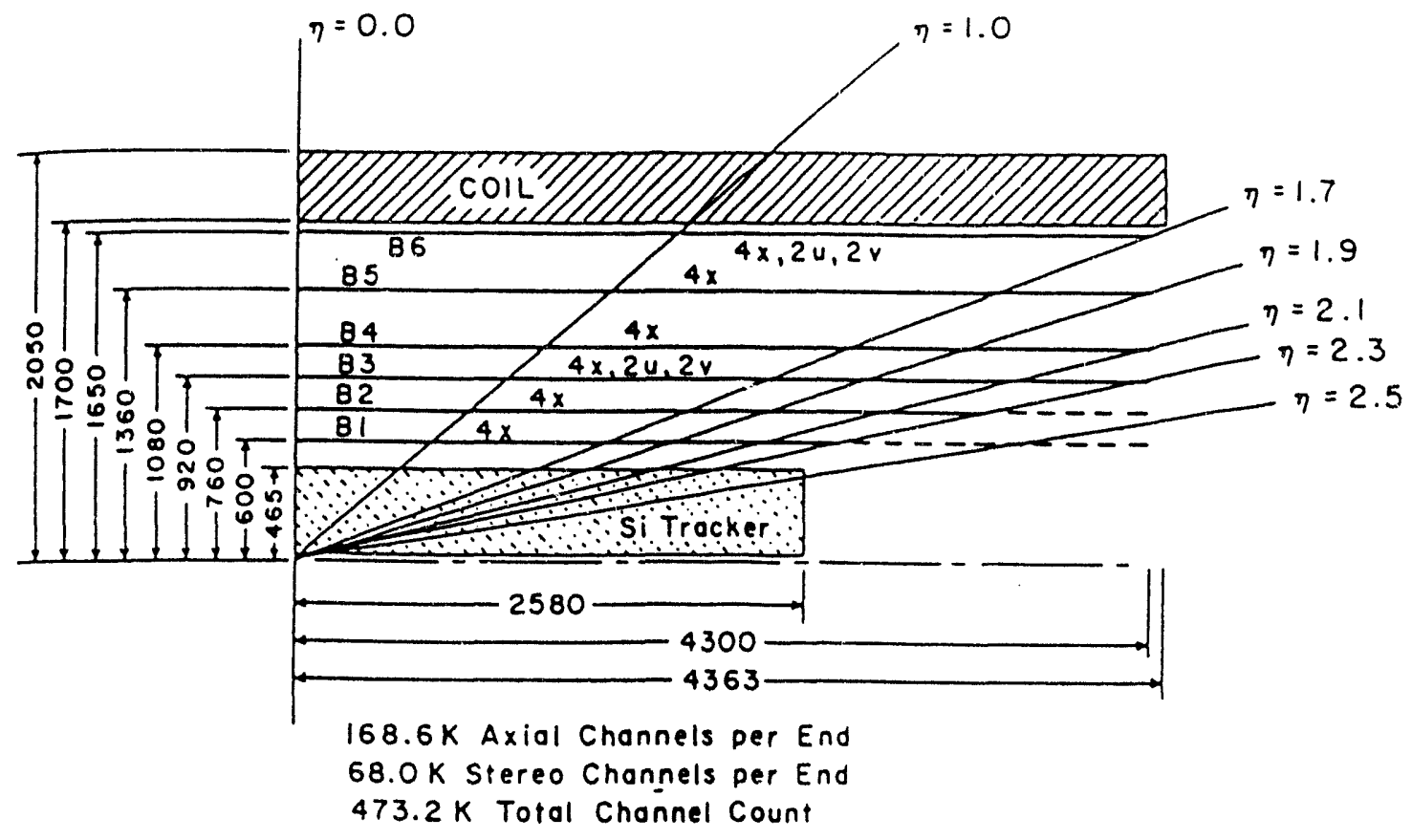

Schematic of the central tracker exhibiting the fiber superlayers.

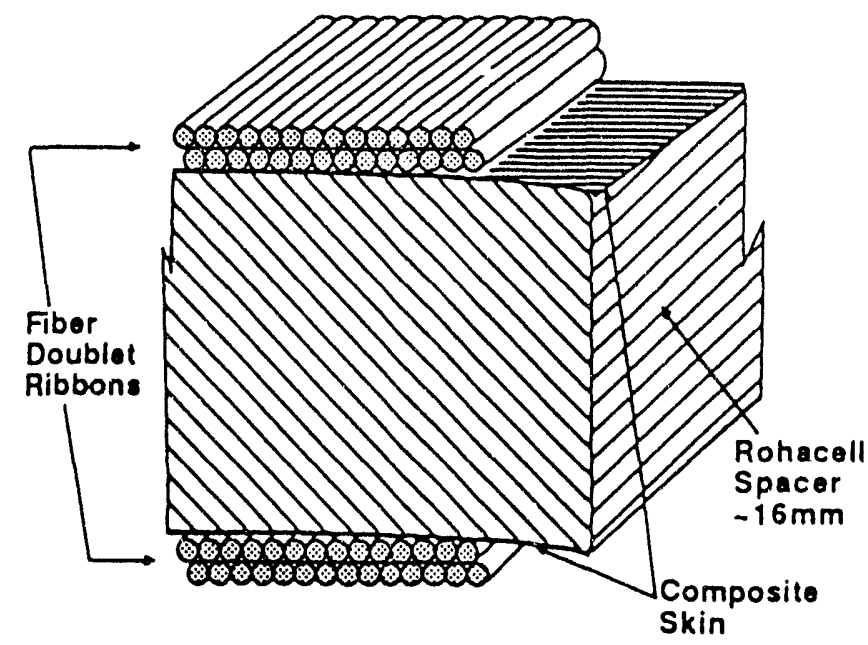

Schematic of a superlayer having axial fiber doublet ribbons mounted on the inner and outer surfaces of a support cylinder.

Figure 1 

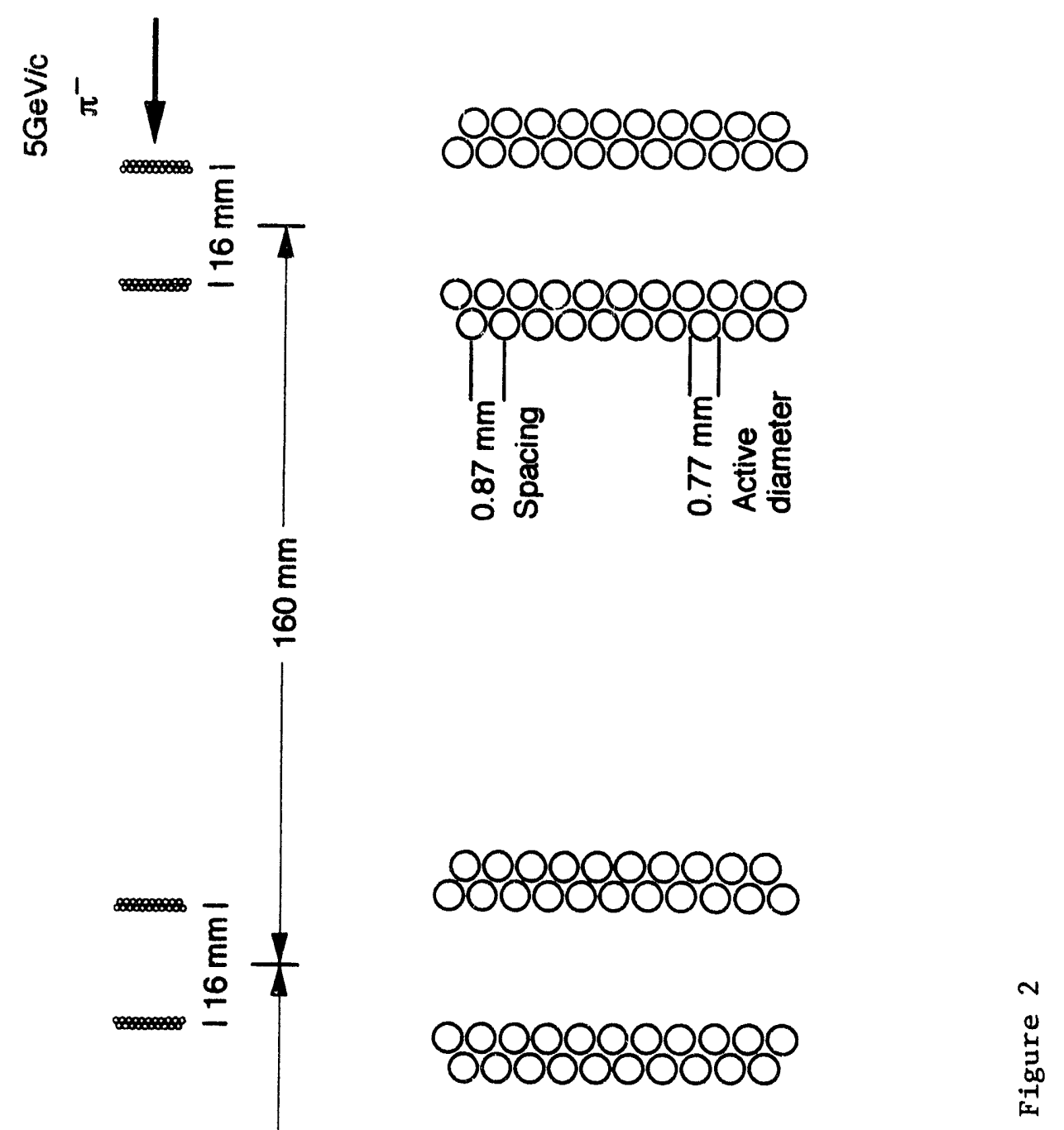


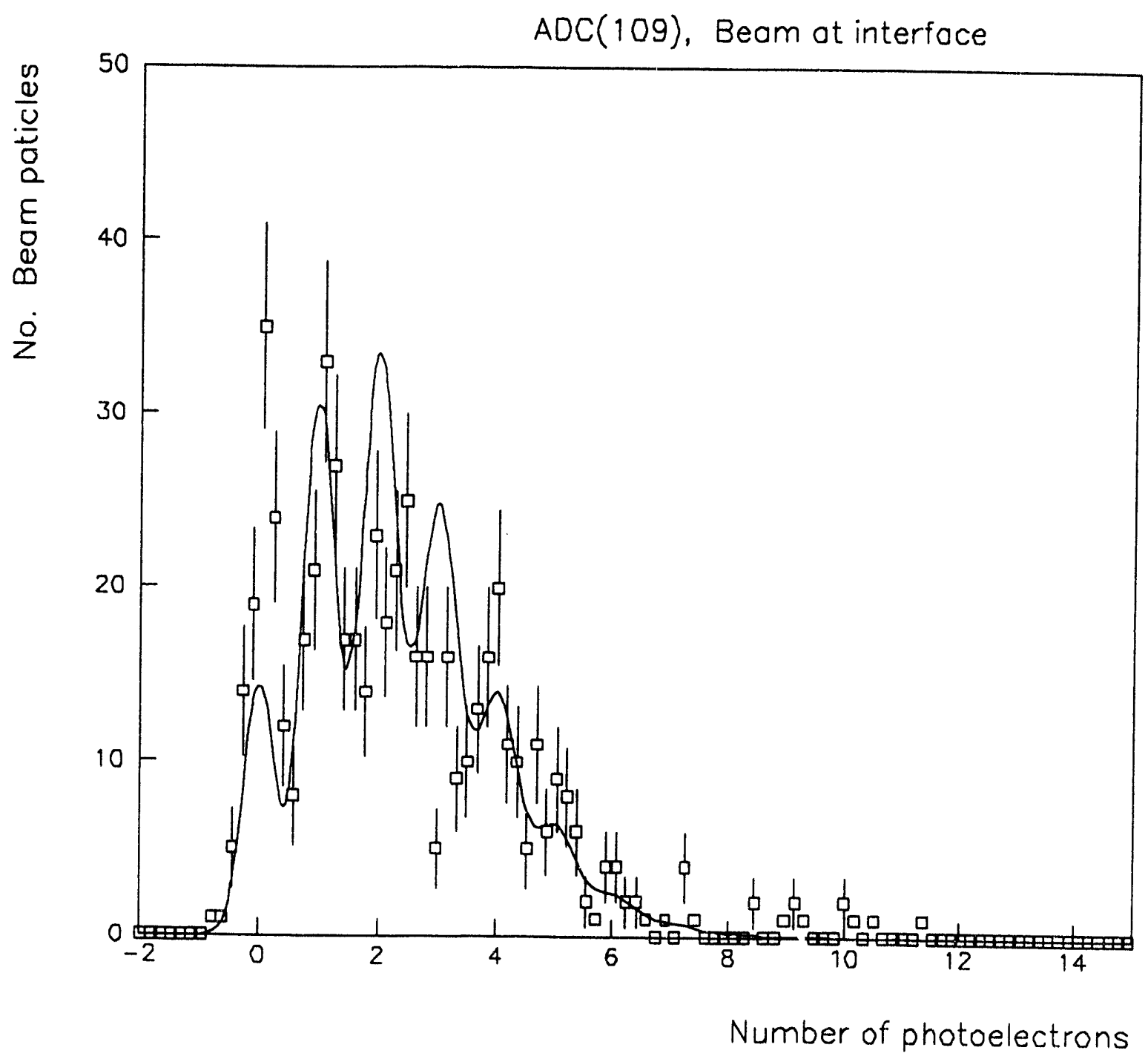

Figure 3 


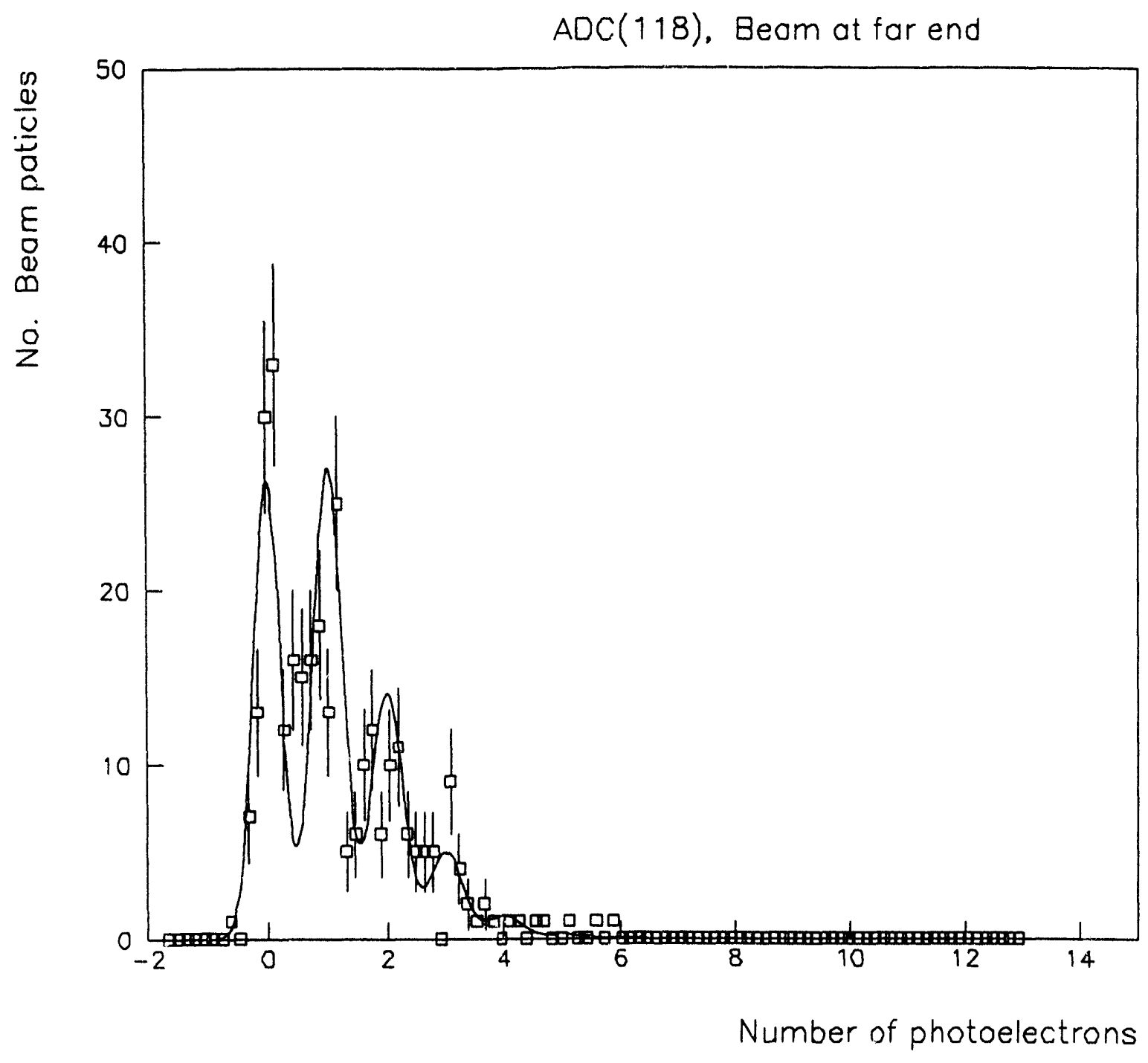

Figure 4

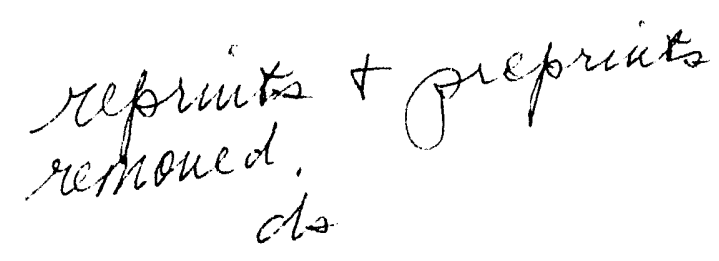



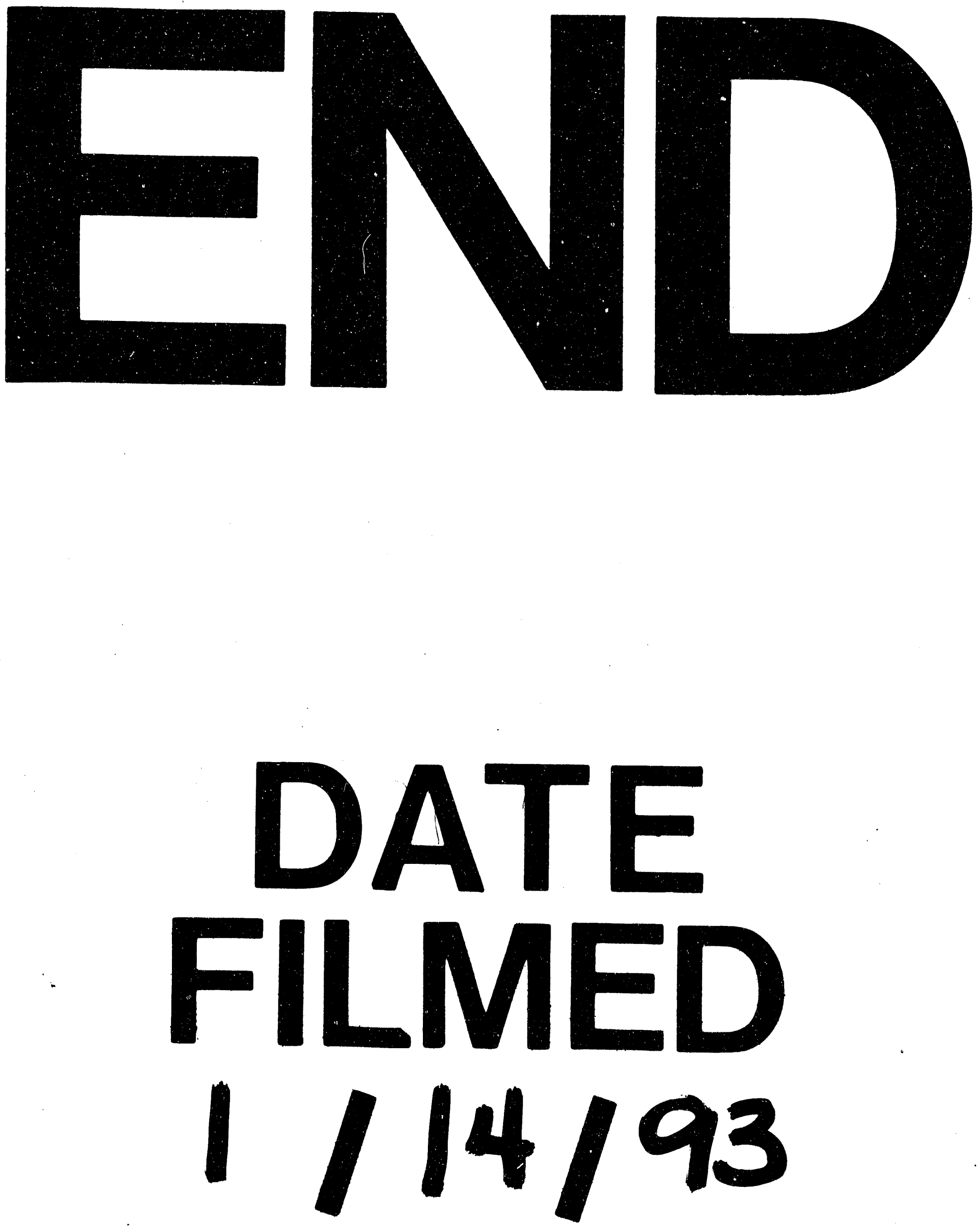
